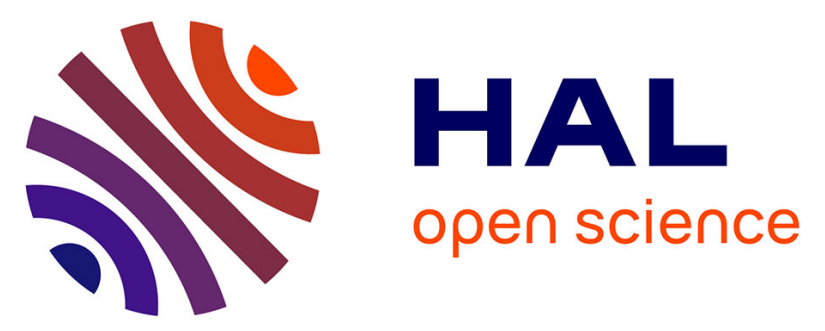

\title{
Bacterial gut dysbiosis is associated with Crohn's disease symptoms but not with elevated fecal calprotectin
}

Sylvie Buffet-Bataillon, Céline Landreau, Laurent Siproudhis, Vincent Cattoir, Guillaume Bouguen

\section{- To cite this version:}

Sylvie Buffet-Bataillon, Céline Landreau, Laurent Siproudhis, Vincent Cattoir, Guillaume Bouguen. Bacterial gut dysbiosis is associated with Crohn's disease symptoms but not with elevated fecal calprotectin. Clinics and Research in Hepatology and Gastroenterology, 2021, 45 (2), pp.101669. 10.1016/j.clinre.2021.101669 . hal-03190699

\section{HAL Id: hal-03190699 \\ https://hal.science/hal-03190699}

Submitted on 21 Apr 2021

HAL is a multi-disciplinary open access archive for the deposit and dissemination of scientific research documents, whether they are published or not. The documents may come from teaching and research institutions in France or abroad, or from public or private research centers.
L'archive ouverte pluridisciplinaire HAL, est destinée au dépôt et à la diffusion de documents scientifiques de niveau recherche, publiés ou non, émanant des établissements d'enseignement et de recherche français ou étrangers, des laboratoires publics ou privés. 


\section{MANUSCRIPT RESEARCH LETTER}

Title : Bacterial gut dysbiosis is associated with Crohn's disease symptoms but not with elevated fecal calprotectin.

Sylvie Buffet-Bataillon ${ }^{a}$, Clémence Landreau ${ }^{a}$, Laurent Siproudhis ${ }^{c}$, Vincent Cattoir ${ }^{b}$, Guillaume Bouguenc

${ }^{a}$ CHU Rennes, Univ Rennes, INSERM, Institut NUMECAN (Nutrition Metabolisms and Cancer), F-35000 Rennes, France

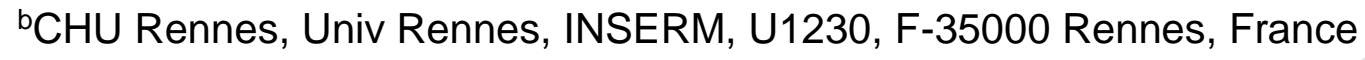

'CHU Rennes, Univ Rennes, INSERM, CIC1414, Institut NUMECAN (Nutrition

Metabolisms and Cancer), F-35000 Rennes, France

\section{Corresponding author:}

Department of Bacteriology, CHU de Rennes,

2 rue Henri Le Guilloux, 35033 Rennes, France,

Email address: sylvie.buffet-bataillon@chu-rennes.fr. (S. Buffet-Bataillon)

\section{Highlights:}

- What is already known on this subject?

The relationship between the gut microbiota and Crohn's disease is clearly establish.

- What are the new findings?

A distinct bacterial dysbiosis has been observed in patients with symptomatic Crohn's disease, independent of mucosal inflammation.

- How might it impact on clinical practice in the foreseeable future?

The treatment of gut dysbiosis in Crohn's disease patient may improve their symptoms.

Key words: Crohn's disease, Microbiota, Fecal calprotectin, Symptoms, Mucosal 
inflammation, Probiotic.

In inflammatory bowel diseases (IBDs), the global composition of the gut microbiota contains specific pathogens that may be relevant to the etiology and pathogenesis of the disease. Predominant changes described in the literature regarding the gut microbiota composition of individuals with Crohn disease (CD), include: alterations in the proportion of Bacteroidetes and Firmicutes, an increase in the percentage of Gammaproteobacteria and Enterobacterales (i.e. Escherichia coli in CD with ileal disease), and a decrease in Clostridiales (i.e. Faecalibacterium prausnitzii) [1,2]. Furthermore, in the paper by Sokol et al., besides bacterial dysbiosis, their study identified a distinct fungal microbiota during IBD characterized by alterations in biodiversity and composition that might play a role in IBD pathogenesis [3]. They also observed a decrease of the alpha diversity in CD, particularly in samples from flaring patients. However, the disease activity was assessed by physicians based on symptoms without objective markers of inflammation, such as fecal calprotectin (FC). A known discrepancy exists between symptoms and mucosal inflammation during CD, thus making difficult to diagnose a flare only based on symptoms [4]. Of note, irritable bowel syndrome has also been associated with a reduction of microbial diversity [5]. This remains unclear whether the decrease of bacterial diversity during a flare of $C D$ is linked to the symptoms or the mucosal inflammation. The aim of the study was then to compare the gastrointestinal tract microbiota in $C D$ patients during flare of $C D$ and/or mucosal inflammation.

Stool samples from 125 consecutive adult patients with a confirmed diagnosis of CD were collected during a one-year period at the referral center Hospital University of Rennes (France). The Harvey Bradshaw (HB) index was assessed at each time of a stool collection. CD patients were grouped for dichotomous analysis with an HB score 
of $\geq 5$ considered as symptomatic $\mathrm{CD}$ and those with an $\mathrm{HB}$ score $<5$ as asymptomatic $\mathrm{CD}$ [6]. FC levels are representative of neutrophil migration into the intestinal mucosa that occurs in the process of intestinal inflammation, and in clinical practice, they are commonly used as a non-invasive biomarker that significantly correlates with inflammatory disease activity and response to therapy [7]. The FC level was measured for each sample using a fully automated chemiluminescent immunoassay (LIAISON Calprotectin, DiaSorin). The absence of mucosal inflammation was defined with a fecal calprotectin $(\mathrm{FC})$ value $<50 \mu \mathrm{g} / \mathrm{g}$. According to the International Human Microbiome Standards (IHMS), patients were asked to collect fecal samples immediately after defecation in a sterile container (VWR). Then, the stool samples were stored up to 24 hours at $4^{\circ} \mathrm{C}$. When received at the laboratory, all samples were manually homogenized and weighed into separate aliquots for storage at $-80^{\circ} \mathrm{C}$ until DNA extraction. The composition of microbial populations was assessed by 16S rRNA gene sequence-based microbiota analysis. DNA was extracted from fecal specimens with the automated MagnaPure (Roche Diagnostics $\mathrm{GmbH}$, Mannheim, Germany) instrument, using the MagNA Pure LC DNA Kit III (bacteria, fungi) (Roche) according to the manufacturer's recommendations. Primers were designed according to the V3V4 regions of the bacterial $16 \mathrm{~S}$ rRNA (the upstream primer: PCR_341 $\mathrm{F}$ is 5'CCTACGGGNGGCWGCAG-3', and the downstream primer: PCR_785R is 5'GACTACHVGGGTATCTAATCC-3') [8]. The primers were linked to Illumina sequencing adapters and were used in PCR amplification; the PCR products were purified, quantified, and then normalized to form a sequencing library. These libraries first were underwent quality control and qualified libraries were sequenced by Illumina MiSeq-Using paired 250-bp reads as outlined in the Illumina 16 S sample preparation guide [9]. The raw image data files obtained from the Illumina MiSeq were analyzed 
and transformed into raw sequence reads by base calling, and the results were stored as FASTQ files, containing sequence information of reads and their corresponding sequencing qualities. The individual sequence reads obtained were filtered, trimmed and processed as described by Escudie and al. according to Find Rapidly OTUs with Galaxy Solution (FROGS) [10]. All reads were classified to the lowest possible taxonomic rank using FROGS and a reference dataset from the SILVA 16S database (https://www.arb-silva.de/download/arb-files/). A table of 16S rRNA Operational Taxonomic Units (OTUs) and an evolutionary tree were then generated while the $\mathrm{R}$ software (version 3.5.1) was used for all analyses. Descriptive statistics and visualization of the microbiome data were conducted in $\mathrm{R}$ using multiple packages, including 'phyloseq', 'microbiome', 'reshape2','ggplot2', 'grid', 'ape', 'scales', 'DESeq2','vegan', 'corrplot', 'RCy3', 'igraph' [11]. The Kruskal-Wallis test was applied to compare global composition of bacterial microbiota differences at the phylum level. The Chao1 and Shannon indices were used to assess alpha diversity. The Bray-Curtis dissimilarity matrix was used to determined beta diversity and visualized using principal coordinates analysis (PCOA) according to a variant of PERMANOVA procedure ("adonis" function in the R "vegan" package). To determine species associated with patients' symptoms, the differential abundance of bacterial species between patients was determined using DESeq2 with $p$-value adjusted for multiple testing using the Benjamini-Hochberg method (cut-off $=$ adjusted $p<0.05$ ). Positive log-fold change pointed out an increase in abundance, while negative log-fold change pointed out a reduction in abundance in the symptomatic patients compared to asymptomatic patients. To investigate the interactions and differences of OTUs in the two groups of patients, we computed the Pearson correlations and drew the correlation matrix and 
interaction network with Cystoscape (https://cytoscape.org/). We used the False Discovery rate (FDR) method to adjust $p$-values for multiple testing.

A total of fecal microbiota from $125 \mathrm{CD}$ patients, including: 25 symptomatic patients ( $\mathrm{HB}$ index $\geq 5$ ) with a concomitant value of $\mathrm{FC} \geq 50 \mu \mathrm{g} / \mathrm{g} ; 17$ symptomatic patients (HB 25) with no mucosal inflammation ( $\mathrm{FC}<50 \mu \mathrm{g} / \mathrm{g}) ; 59$ asymptomatic patients $(\mathrm{HB}<5)$ with a concomitant value of $\mathrm{FC} \geq 50 \mu \mathrm{g} / \mathrm{g} ; 24$ asymptomatic patients $(\mathrm{HB}<5)$ with no mucosal inflammation ( $F C<50 \mu \mathrm{g} / \mathrm{g}$ ). Details regarding age, female sex, Body Mass Index $(\mathrm{BMI})>25 \mathrm{~kg} / \mathrm{m} 2$, smoking, disease duration, gastrointestinal surgery, Montreal A, B, L, fecal calprotectin $(\mu \mathrm{g} / \mathrm{g}$ ), treatment within 90 days of microbiota analysis (AntiTNF, Thiopurine-methotrexate (MTX)) are shown in supplementary Table 1. The global composition of bacterial microbiota at the phyla level in patients with symptoms showed lower relative abundance of Firmicutes and Bacteroidetes while the phyla Proteobacteria and Fusobacteria were more represented, independently of the FC level (i.e., $<$ or $\geq 50 \mu \mathrm{g} / \mathrm{g}$ ) (Figure 1). Interestingly, we observed no alteration of the abundance of phyla for asymptomatic CD patients ( $\mathrm{HB} \leq 4)$ even with $\mathrm{FC}$ value $\geq 50$ $\mu \mathrm{g} / \mathrm{g}(\mathrm{p}<0.05)$. The comparison of microbial community structure using alpha diversity indices showed a lower number of detected species (Chao1) as well as a lower diversity (Shannon) in symptomatic patients as compared to asymptomatic patients $(p=0.02)$. Symptomatic and asymptomatic patients also differed according to the community structures (PERMANOVA, $\mathrm{p}=0.002$ ) as well as the differential abundance analysis of the species-level taxonomic assignments regardless the FC level. The abundance of several taxa was decreased in case of symptoms, particularly within the phylum Firmicutes. The abundances of genera Escherichia-Shigella, Enterococcus, Streptococcus, Megasphaera, Fusobacterium, Erysipelatoclostridium, Butyricicoccus, Gemella, Prevotella, Solobacterium, Lactobacillus, Akkermansia were significantly 
enriched in the microbiota of symptomatic patients $(p<0.05)$ (Figure $1 B)$. The changes in Escherichia-Shigella and Streptococcus proportions were particularly remarkable for all symptomatic patients. We further investigated the existence of correlations at the genus level according to symptoms (Figure $1 \mathrm{C}$ ). Symptomatic patients were more likely to present a positive correlation between the abundance of Faecalibacterium and Bacteroides $\left(p=4.510^{-7}\right)$ and Subdoligranulum $\left(p=4.510^{-6}\right)$; Ruminococcus torques group and Blautia $\left(\mathrm{p}=4.510^{-6}\right)$ and Alistipes and Ruminococcaceae UGC-002 $\left(\mathrm{p}=7.710^{-}\right.$ 3) (Figure $1 \mathrm{C} 1$ ). In asymptomatic patients, a positive correlation was found between the abundance of Escherichia-Shigella and Lachnoclostridium $\left(\mathrm{p}=2.710^{-4}\right)$; Streptococcus and Veillonella $\left(\mathrm{p}=1.610^{-6}\right)$; Ruminoclostridium 5 and Ruminoccocus torques group $\left(\mathrm{p}=1.610^{-7}\right)$ and Ruminococcaceae UCG-002 $\left(\mathrm{p}=1.510^{-8}\right)$ (Figure $\left.1 \mathrm{C} 2\right)$. Similar results were obtained with interaction network generated using Cytoscape (data not shown) and were not affected by the absence of mucosal inflammation or not.

To our knowledge, this is the first study assessing the microbiota in patients with flare and/or FC concentration $<$ or $\geq 50 \mu \mathrm{g} / \mathrm{g}$. These data underlines a distinct bacterial microbiota dysbiosis in symptomatic patients independently of the FC level. Indeed, dysbiosis was much more predominant in symptomatic patients than in asymptomatic patients with mucosal inflammation. The differential abundance of species between patients allowed us determining species signatures of symptomatic CD. EscherichiaShigella, Fusobacterium, Gemella and Streptococcus were overrepresented in symptomatic patients. Moreover, Ruminococcus 1, Lachnospira, Clostridium sensu stricto 1 and Ruminococcus 2 were also underrepresented in symptomatic patients. The proportions of these OTUs were already described as altered in CD [12,13]. However, as those articles focused on healthy controls, they cannot be related to the 
presence of symptoms. The presence of Escherichia-Shigella and Streptococcus were identified in all symptomatic patients. So, these "key microbial signatures" would help with the diagnosis of symptomatic CD. The interaction network showed that the interactions and differences of OTUs were different in the two groups of patients. Some interactions were specific to symptomatic $C D$ and others to asymptomatic $C D$. In asymptomatic CD, Escherichia-Shigella and Streptococcus were positively correlated with Lachnoclostridium and Veillonella, respectively. However, for symptomatic CD, Escherichia-Shigella and Streptococcus had no correlation with any other OTUs. This lack of correlation may be related to symptoms in CD patients. Furthermore, the interaction network results need to be taken into consideration when creating probiotics combination to treat CD. For instance, the probiotic combination VSL\#3 (a probiotic preparation of eight live freeze-dried bacterial species, including Lactobacillus casei, L. delbrueckii subsp. bulgaricus, L. acidophilus, L. plantarum, Bifidobacterium longum, B. infantis, B. breve, and Streptococcus salivarius subsp. thermophilus) and E. coli Nissle, which do not take under consideration this interaction network, were shown to reduce active inflammation and sustain remission only in ulcerative colitis (UC) but not in CD $[14,15]$. Some limitations regarding this study should be acknowledged. The patient populations according to being symptomatic or not were broadly comparable. However, symptomatic patients without mucosal inflammation correspond to patients with older diseases or history of surgery. In conclusion, this study suggests a possible association between gut dysbiosis and the presence or absence of Crohn's disease symptoms, independently of fecal calprotectin levels. These microbial signatures linked to symptoms may be taken into account when planning to evaluate the efficacy of probiotics or fecal microbiota transplantation in the treatment of Crohn's disease. 


\section{Disclosure of interest}

The authors declare that they have no competing interest.

\section{Declaration of interests}

$\otimes$ The authors declare that they have no known competing financial interests or personal relationships that could have appeared to influence the work reported in this paper.

\section{Acknowledgment}

None. 


\section{References}

1. Kostic AD, Xavier RJ, Gevers D. The microbiome in inflammatory bowel disease: current status and the future ahead. Gastroenterology. 2014;146(6):1489-99.

2. Mcllroy J, laniro G, Mukhopadhya I, Hansen R, Hold GL. Review article: the gut microbiome in inflammatory bowel disease-avenues for microbial management. Aliment Pharmacol Ther. 2018;47(1):26-42.

3. Sokol H, Leducq V, Aschard H, Pham HP, Jegou S, Landman C, et al. Fungal microbiota dysbiosis in IBD. Gut. 2017;66(6):1039-1048.

4. Peyrin-Biroulet L, Reinisch W, Colombel JF, Mantzaris GJ, Kornbluth A, Diamond $\mathrm{R}$,et al. Clinical disease activity, C-reactive protein normalisation and mucosal healing in Crohn's disease in the SONIC trial. Gut. 2014;63(1):88-95.

5. Lo Presti A, Zorzi F, Del Chierico F, Altomare A, Cocca S, Avola A, et al. Fecal and Mucosal Microbiota Profiling in Irritable Bowel Syndrome and Inflammatory Bowel Disease, Front Microbiol. 2019;10:1655.

6. Vermeire S, Schreiber S, Sandborn WJ, Dubois C, Rutgeerts P. Correlation between the Crohn's disease activity and Harvey- Bradshaw indices in assessing Crohn's disease severity. Clin Gastroenterol Hepatol. 2010;8(4):357-63.

7. Smith LA, Gaya DR. Utility of faecal calprotectin analysis in adult inflammatory bowel disease. World J Gastroenterol. 2012;18(46):6782-6789.

8. Klindworth A, Pruesse E, Schweer T, Peplies J, Quast C, Horn M, et al. Evaluation of general $16 \mathrm{~S}$ ribosomal RNA gene PCR primers for classical and next-generation sequencing-based diversity studies. Nucleic Acids Res. 2013 Jan 7;41(1):e1.

9. Illumina, 16S sample preparation guide, 2016. http:// www.illumina.com/content/dam/illumina-support/

documents/documentation/chemistry_documentation/ 16s/16s-metagenomic-library- 
prepguide- 15044223-b. pdf.

10. Escudié F, Auer L, Bernard M, Mariadassou M, Cauquil L, Vidal K, et al. FROGS: Find, Rapidly, OTUs with Galaxy Solution. Bioinformatics. 2018;34(8):1287-1294.

11. McMurdie PJ, Holmes S. phyloseq: an R package for reproducible interactive analysis and graphics of microbiome census data. PLoS One. 2013;8(4):e61217.

12. Forbes JD, Chen CY, Knox NC, Marrie RA, El-Gabalawy H, de Kievit T, et al. A comparative study of the gut microbiota in immune mediated inflammatory diseasesdoes a common dysbiosis exist? Microbiome. 2018;6(1):221.

13. Pascal V, Pozuelo M, Borruel N, Casellas F, Campos D, Santiago A, et al. A microbial signature for Crohn's disease. Gut. 2017;66(5):813-822.

14. Bibiloni R, Fedorak RN, Tannock GW, Madsen KL, Gionchetti P, Campieri M,et al. VSL\# 3 probiotic-mixture induces remission in patients with active ulcerative colitis. Am J Gastroenterol. 2005;100(7):1539-46.

15. Schultz M. Clinical use of E. coli Nissle 1917 in inflammatory bowel disease. Inflamm. Inflamm Bowel Dis. 2008;14(7):1012-8. 


\section{FIGURE LEGENDS}

\section{Figure 1.}

A. Global composition of bacterial microbiota at the phyla level. Symptomatic CD was defined according to the Harvey Bradshaw Index $(\mathrm{HB}) \geq 5$.

B. Significant $(p<0.05)$ log-fold changes in the abundances of bacterial species in symptomatic patients.

C. Correlation matrix between bacterial groups in symptomatic patients (C1) and asymptomatic patients (C2). Positives values (green rounds) indicate positive correlations, and negative values (red rounds) indicate inverse correlations. The shading of the round indicates the magnitude of the association; darker rounds are more strongly associated than lighter rounds. The sign of the correlation was determined using Spearman's method. 
Figure 1. A

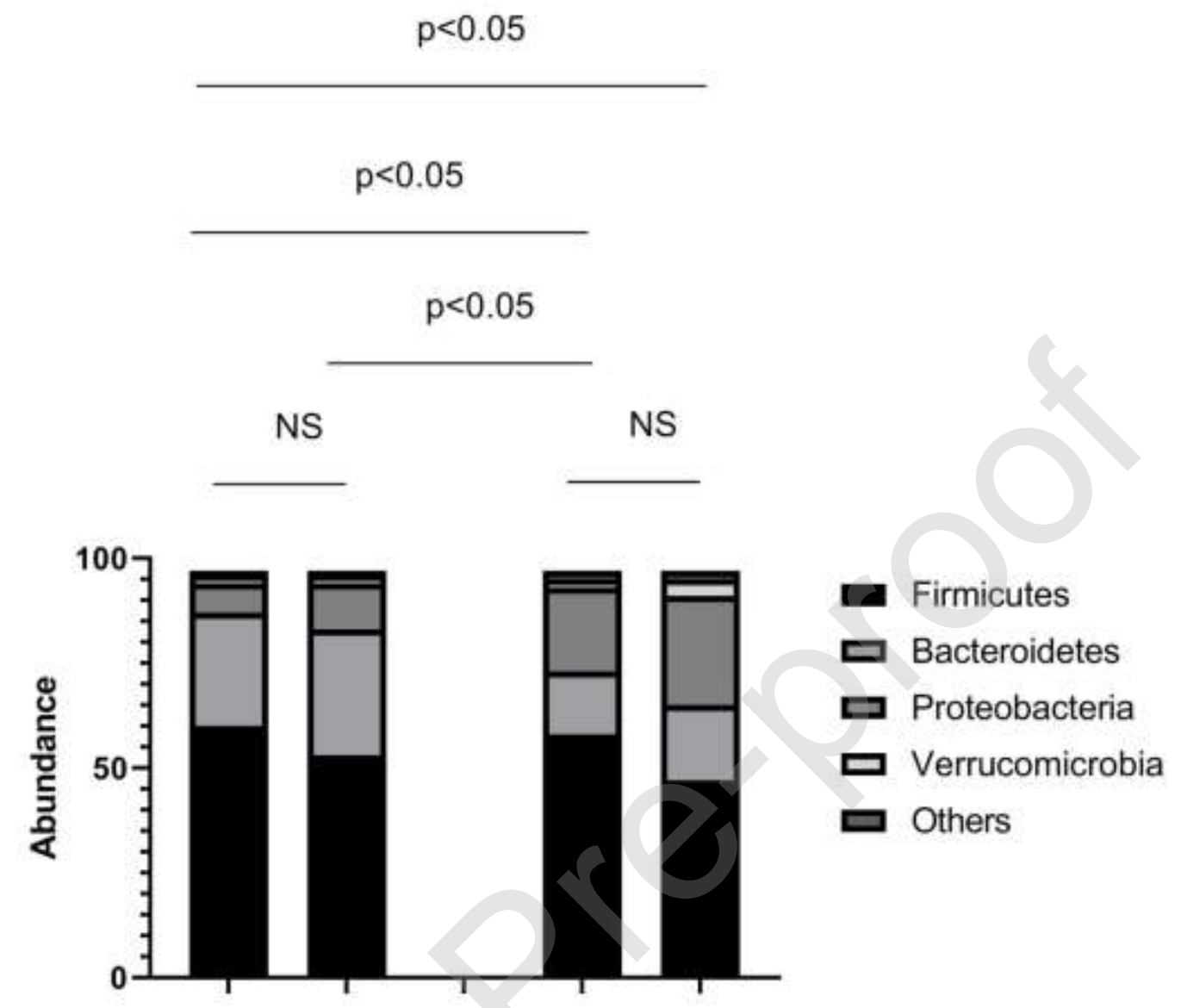

Symptomatic CD

No No

Yes Yes

Level of fecal calprotectin $\geq 50$

$<50$

$<50 \geq 50$

$(\mu \mathrm{g} / \mathrm{g})$ 


\section{Figure 1. B}

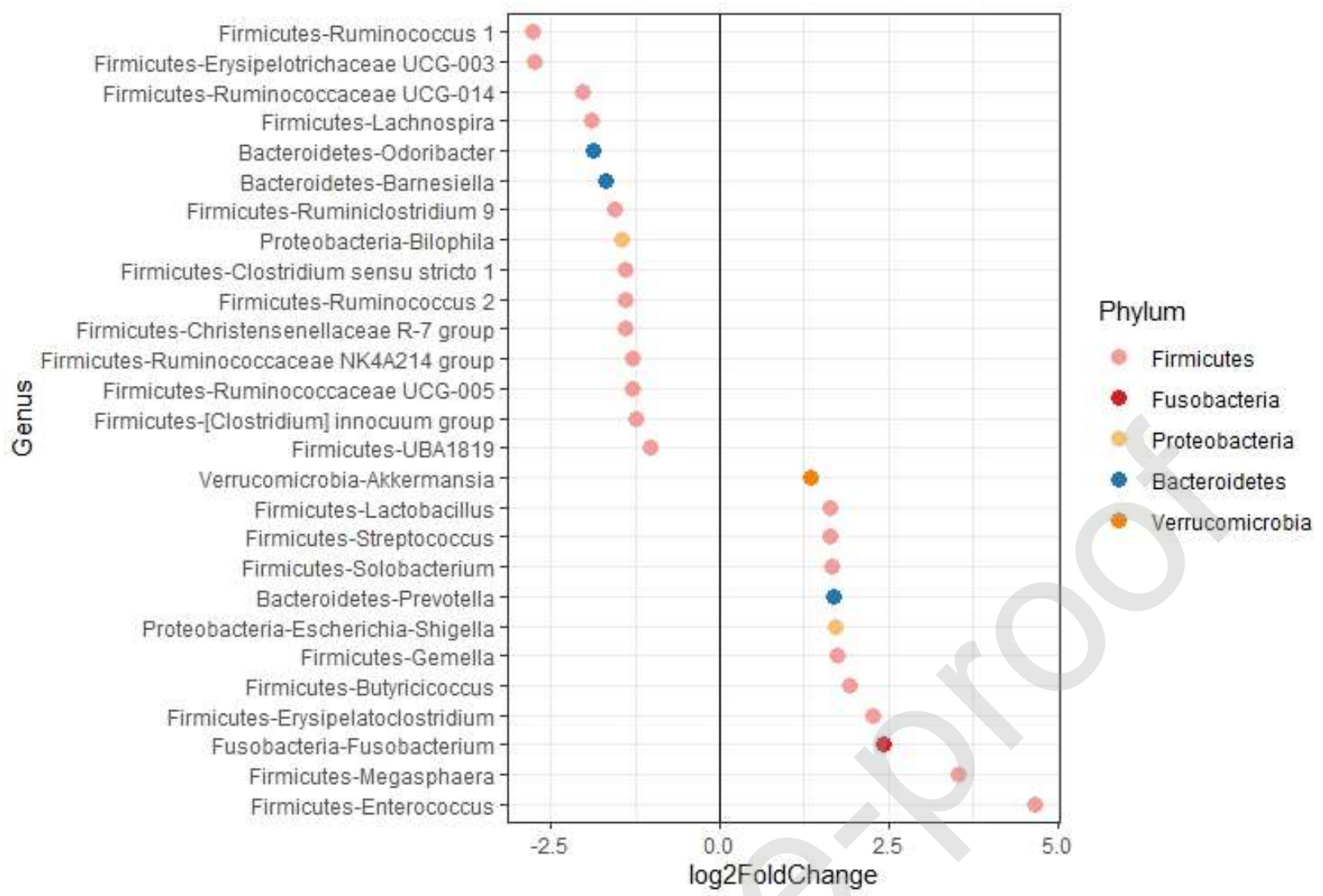




\section{Figure 1. C1}

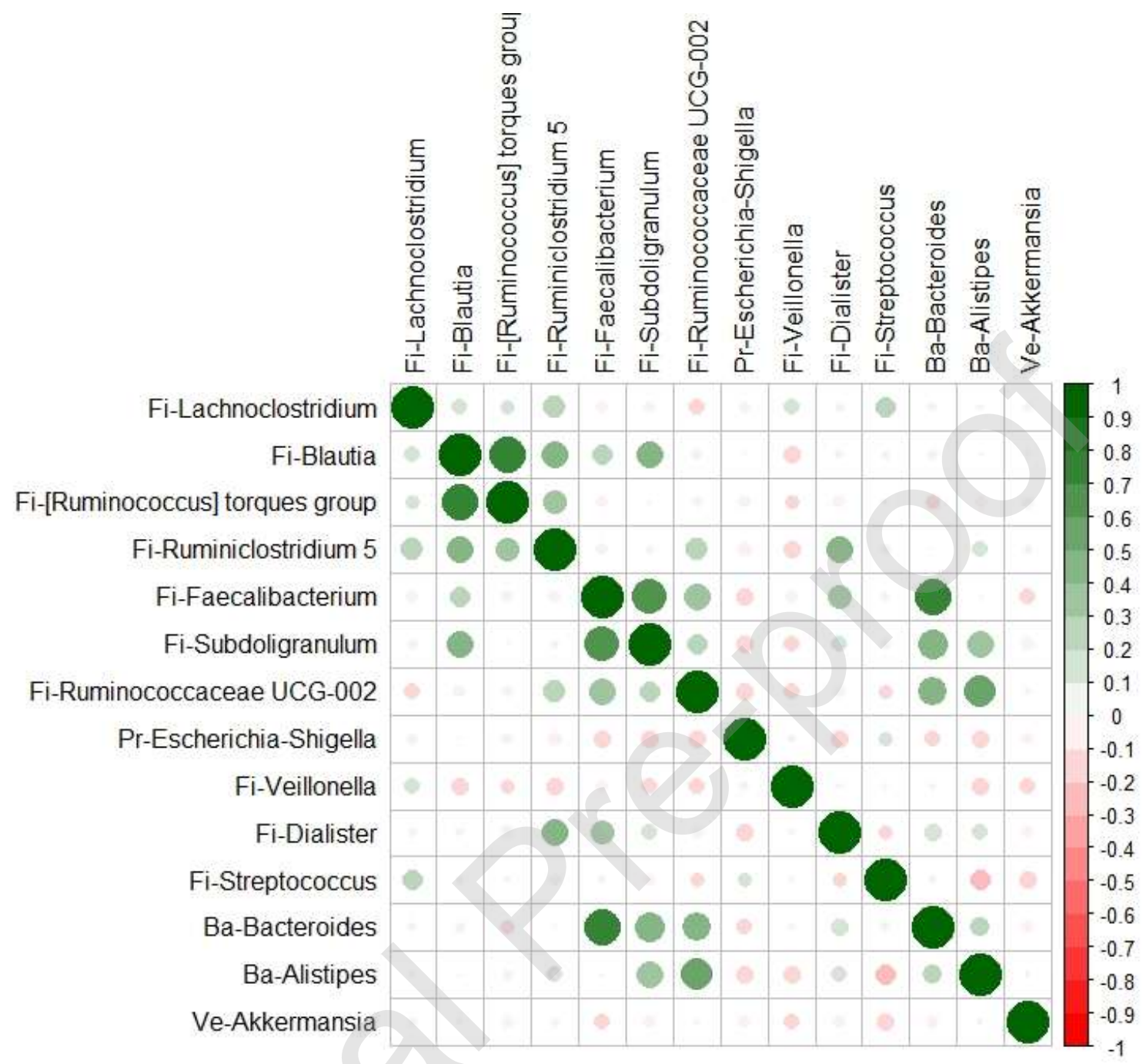




\section{Figure 1. C2}

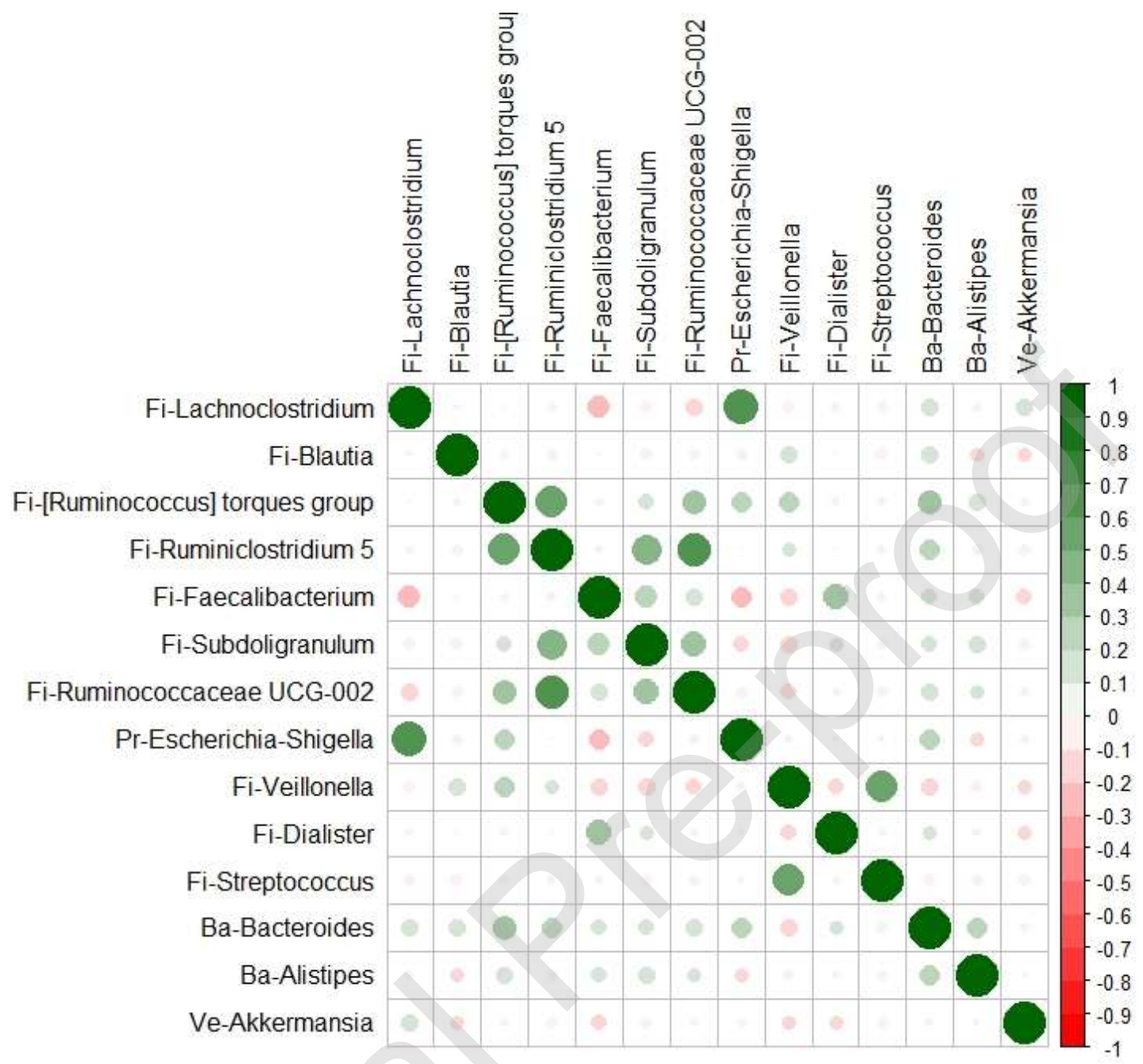

